\title{
Identification and Characterization of Botrytis fragariae Isolates on Strawberry in the United States
}

Madeline E. Dowling, Meng-Jun Hu, and Guido Schnabel, ${ }^{\dagger}$ Department of Plant and Environmental Sciences, Clemson University, Clemson, SC 29634

\begin{abstract}
Gray mold is a devastating disease on strawberry, and may be caused by several species of Botrytis. The goal of this study was to better understand and characterize the species of Botrytis with reduced sensitivity to the fungicide Polyoxin D, particularly Botrytis fragariae. In total, 78 Botrytis isolates of unknown species that were sensitive (28 isolates; S), moderately sensitive (22 isolates; MS), or reduced sensitive ( 28 isolates; RS) to Polyoxin-D were collected from commercial strawberry fields of five states in the United States, identified to the species level, and characterized. The majority (75\%) of S isolates were Botrytis cinerea and the majority (79\%) of RS isolates were the recently described species $B$. fragariae, indicating an innate ability of $B$. fragariae to tolerate Polyoxin-D. B. fragariae produced fluffy, white mycelium and was less likely to sporulate on potato

dextrose agar than $B$. cinerea. Isolates from a commercial field recovered from blossoms in early spring were all $B$. fragariae, those from leaves of the same plants in late spring were a mixture of $B$. fragariae and $B$. cinerea, and those from fruit in early summer were all $B$. cinerea, indicating that $B$. fragariae may preferentially colonize blossom tissue. A polymerase chain reaction-based assay was developed based on $N E P 2$ sequence variability to distinguish $B$. fragariae from other Botrytis spp. that have been reported on strawberry, including B. cinerea, B. mali, B. caroliniana, and $B$. ricini. None of the isolates collected from Canada, California, or North Carolina nurseries were $B$. fragariae, indicating that the newly described species may not exist or not be widely distributed in planting stock.
\end{abstract}

The genus Botrytis consists of over 30 plant-pathogenic species with a wide variety of life history traits (Fillinger and Elad 2015). Differentiation of these species was originally hindered because the internal transcribed spacer (ITS) regions commonly used for fungal phylogenies lacked the necessary interspecific variation to distinguish Botrytis spp. More species were delimited after 2005, when the glyceraldehyde 3-phosphate dehydrogenase (G3PDH), RPB2, and HSP60 regions were first used to genetically characterize Botrytis spp., and, in 2007, the NEP1 and NEP2 genes were added (Staats et al. 2005, 2007). The genus Botrytis is now known to be made up of two clades, with clade 1 containing pathogens that infect mostly eudicots whereas clade 2 pathogens infect mostly monocots. Though the genus Botrytis has vast interspecific genetic diversity, many species are difficult to distinguish morphologically (Fillinger and Elad 2015; Fournier et al. 2005; Walker et al. 2011). Because host ranges of species may overlap, it is sometimes necessary to genetically distinguish isolates for accurate data assessment (Chilvers and du Toit 2006), especially when species have different traits such as fungicide resistance, reproduction mechanism, and lifecycle (Fournier et al. 2005).

One common disease caused by several Botrytis spp. is gray mold of strawberry (Li et al. 2012; Plesken et al. 2015). Botrytis cinerea was long considered the sole causal agent of this disease but several species may be involved (Amiri et al. 2016; Li et al. 2012; Plesken et al. 2015). Most of these species have not been detected in the southeastern United States or, like B. caroliniana, B. mali, and B. ricini, do not appear to be widespread (Amiri et al. 2016; Dowling and Schnabel 2017; Li et al. 2012). The newly described species, B. fragariae, appears to be more widespread on strawberry plants and has already been detected in Germany and South Carolina (Rupp et al. 2017). In Germany, where most of the research involving this pathogen has thus far occurred, 38 isolates were found in nine fields and four different states. Unlike B. cinerea, $B$. fragariae appears to be host specific for

\section{${ }^{\dagger}$ Corresponding author. E-mail: schnabe@clemson.edu}

*The $\boldsymbol{e}$-Xtra logo stands for "electronic extra" and indicates that one supplementary file is published online.

Accepted for publication 26 May 2017.

() 2017 The American Phytopathological Society strawberry and there is even evidence of tissue preference (Rupp et al. 2017).

As part of a regional resistance monitoring program, we recently detected Botrytis isolates with reduced sensitivity to the fungicide Polyoxin-D. These isolates were collected from fields that had never been treated with this fungicide or fungicides of similar mode of action. The isolates exhibited slower growth rates and visibly less spore production compared with $B$. cinerea (Dowling et al. 2016). The goal of the present study was to further characterize these isolates. Specific objectives were to (i) identify the isolates to the species level, (ii) develop a polymerase chain reaction (PCR)-based method to distinguish the new species from others affecting strawberry, (iii) determine potential innate differences in sensitivity to Polyoxin-D between $B$. cinerea and $B$. fragariae, (iv) investigate potential sources of $B$. fragariae inoculum, and (v) gather further evidence for potential host tissue preferences.

\section{Materials and Methods}

Botrytis sample origin and isolation. Botrytis isolates of unknown species were previously collected from blighted strawberry blossoms and fruit of farms from the states Ohio, Maryland, Virginia, North Carolina, and South Carolina to determine sensitivity to the fungicide Polyoxin-D zinc salt (referred to as Polyoxin-D) (Dowling et al. 2016). We revived 78 of these isolates from storage (filter paper) for this study (Table 1). These isolates were not collected randomly but, instead, were selected to form a subset of locations and states with an even representation of three sensitivity phenotypes to Polyoxin-D: sensitive ( $\mathrm{S} ; 28$ isolates), moderately sensitive (MS; 22 isolates), and reduced sensitive (RS; 28 isolates). These categories were established based on the isolates' relative growth on Amresco malt extract agar amended with Polyoxin-D at $5 \mu \mathrm{g} / \mathrm{ml}$ : S isolates did not grow, MS isolates grew less than or equal to $70 \%$ of the control, and RS isolates grew more than $70 \%$ of the control. The isolates were from 20 different farms in 18 counties of five states: Ohio (5 isolates), Maryland (7 isolates), Virginia (1 isolate), North Carolina (13 isolates), and South Carolina (52 isolates) (Table 1).

Isolates from the main nursery stock providers for eastern growers were obtained from Dr. N. Peres, University of Florida, to investigate the source of $B$. fragariae in the strawberry fields we profiled (Table 1). In total, 91 isolates came from nursery strawberry leaf debris at nurseries in North Carolina (28 isolates) and California (35 isolates) in the United States and Ontario ( 2 isolates) and Nova Scotia (26 isolates) in Canada (Table 1).

In 2016, symptomatic strawberry tissues were collected during early bloom and at last harvest from a commercial field in Mullins, 
SC set up in a randomized complete block design with four replicate blocks per treatment to determine a possible correlation between host tissue type and infecting species (Table 1). One treatment consisted of six applications of Polyoxin-D in the form of Ph-D WDG fungicide (Arysta LifeScience North America, LLC, Cary, NC) according to strawberry advisory system recommendations based on weather conditions during the growing season (Montone et al. 2016). No fungicides were applied to the other treatment. From Polyoxin-D-treatment blocks, we obtained three leaf isolates and five fruit isolates. From the untreated blocks, we obtained five leaf and five fruit isolates. In total, 28 blossom isolates were collected prior to fungicide applications, whereas isolates from leaves and fruit were collected thereafter. Single-spore isolates were obtained as described previously (Dowling et al. 2016).

Isolate identification by sequencing. The G3PDH gene region was amplified for isolates having unique culture morphology on potato dextrose agar (PDA) using PCR with the primers $G 3 P D H \_f w$ and G3PDH_rev, as described previously (Plesken et al. 2015; Staats et al. 2005). Products were then sequenced (Arizona State University Core Laboratories) to determine their identity to the species level. The resulting sequences were aligned with a G3PDH sequence of B. fragariae from Germany (accession number KX429703) and a BLAST search was also performed for all sequences to ensure accurate species identification.

Species-specific primer development. G3PDH, HSP60, RPB2, $N E P 1$, and NEP2 gene fragment sequences of the three Botrytis

Table 1. Origin and species frequency for all isolates examined in this study

\begin{tabular}{|c|c|c|c|c|}
\hline \multicolumn{2}{|c|}{ Origin } & \multicolumn{3}{|c|}{ Isolate frequency } \\
\hline Source, location & Host tissue & Botrytis fragariae & B. mali & B. cinerea \\
\hline \multicolumn{5}{|l|}{ Grower farms ${ }^{\mathrm{a}}$} \\
\hline Maryland & Blossoms & 0 & 1 & 6 \\
\hline Virginia & Blossoms & 0 & 0 & 1 \\
\hline \multirow[t]{2}{*}{ North Carolina } & Blossoms & 4 & 0 & 1 \\
\hline & Fruit & 0 & 0 & 8 \\
\hline South Carolina & Blossoms & 34 & 2 & 16 \\
\hline Ohio & Blossoms & 1 & 0 & 4 \\
\hline \multicolumn{5}{|l|}{ Grower farm } \\
\hline \multirow[t]{3}{*}{ Mullins, SC } & Blossoms & 28 & 0 & 0 \\
\hline & Leaves & 6 & 0 & 2 \\
\hline & Fruit & 0 & 0 & 10 \\
\hline \multicolumn{5}{|l|}{ Nursery } \\
\hline North Carolina & Leaves & 0 & 0 & 28 \\
\hline California & Leaves & 0 & 0 & 35 \\
\hline Ontario & Leaves & 0 & 0 & 2 \\
\hline Nova Scotia & Leaves & 0 & 0 & 26 \\
\hline
\end{tabular}

spp. obtained for a previous study (Rupp et al. 2017) were scanned for polymorphism between species that would allow for differentiation using a PCR assay. The NEP2 gene was chosen because it contained multiple regions where nucleotide sequence varied between all three species (Fig. 1). The NEP2 nucleotide sequences of $B$. cinerea, $B$. fragariae, and $B$. mali used to develop these primers are provided in Supplementary File S1. Using the Multiple Primer Analyzer website by Thermo Fisher Scientific, we compared potential primer sequence combinations to ensure that there was no dimer formation between potential primers and that melting temperatures were similar enough to be combined in a single PCR. A forward primer common between species and three species-specific reverse primers were designed to distinguish $B$. cinerea, B. fragariae, and B. mali (Table 2). The primers were initially tested on 15 isolates representing all three species from four states and 13 farms, and results were confirmed by sequencing the $G 3 P D H$ region. Primers were also tested on common contaminants obtained from strawberry fruit and flowers, including Fusarium, Rhizopus, Cladosporium, and Penicillium spp., to determine whether nonspecific amplification of these contaminants was possible. Each PCR consisted of a small amount of fungal hyphae, hot start Taq PCR mix (Bioneer, Alameda, CA), $1 \mu$ l of each primer (20 $\mu \mathrm{M}$ concentration), and $16 \mu \mathrm{l}$ of DNase-free sterile water for a total reaction volume of $20 \mu \mathrm{l}$. The amplification program began with an initial denaturation step of $95^{\circ} \mathrm{C}$ for $3 \mathrm{~min}$, followed by 34 cycles of denaturation at $95^{\circ} \mathrm{C}$ for $30 \mathrm{~s}$, annealing at $55^{\circ} \mathrm{C}$ for $30 \mathrm{~s}$, and extension at $72^{\circ} \mathrm{C}$ for $1 \mathrm{~min}$. This was followed by a final extension step of $72^{\circ} \mathrm{C}$ for $5 \mathrm{~min}$. Gel electrophoresis of PCR products was performed on $1.5 \%$ agarose gels run at $110 \mathrm{~V}$ for 20 to $30 \mathrm{~min}$.

Primers were also tested on Botrytis aerial mycelium growing from inoculated, detached strawberry fruit. Commercially grown strawberry fruit were washed with soap and water, then surface sterilized in a $10 \%$ bleach solution for $1 \mathrm{~min}$. After drying, they were inoculated by removing a plug from the side of each strawberry using a sterile cork-borer approximately $5 \mathrm{~mm}$ in diameter. The same corkborer was used to transfer agar plugs with actively growing mycelium of isolates 28-2-15, 8-3-16, and 65-4-15 of B. cinerea; 5-5-16, 15-1-16, and 43-1-15 of $B$. fragariae; and 55-6-16, 28-6-15, and 49-10-16 of $B$. mali into the fruit opening. Inoculated strawberry fruit were placed in sealable plastic boxes with moistened paper towels, as described previously (Dowling et al. 2016). After 2 to 3 days or when Botrytis mycelium was visible, an amount of hyphae just covering the tip of a toothpick was removed and placed directly into the PCR, as described above. The newly developed primers were then used to screen all isolates not sequenced at the $G 3 P D H$ region, as well as all isolates from nurseries and from Mullins, SC. Mycelium from B. caroliniana and DNA from $B$. ricini isolates was also used as a template for the speciesspecific primers to determine whether any amplicons would be produced.

Statistical analysis. A $t$ test was performed using the software JMP 11.0 (SAS Institute, Inc., Cary, NC) with a significance level

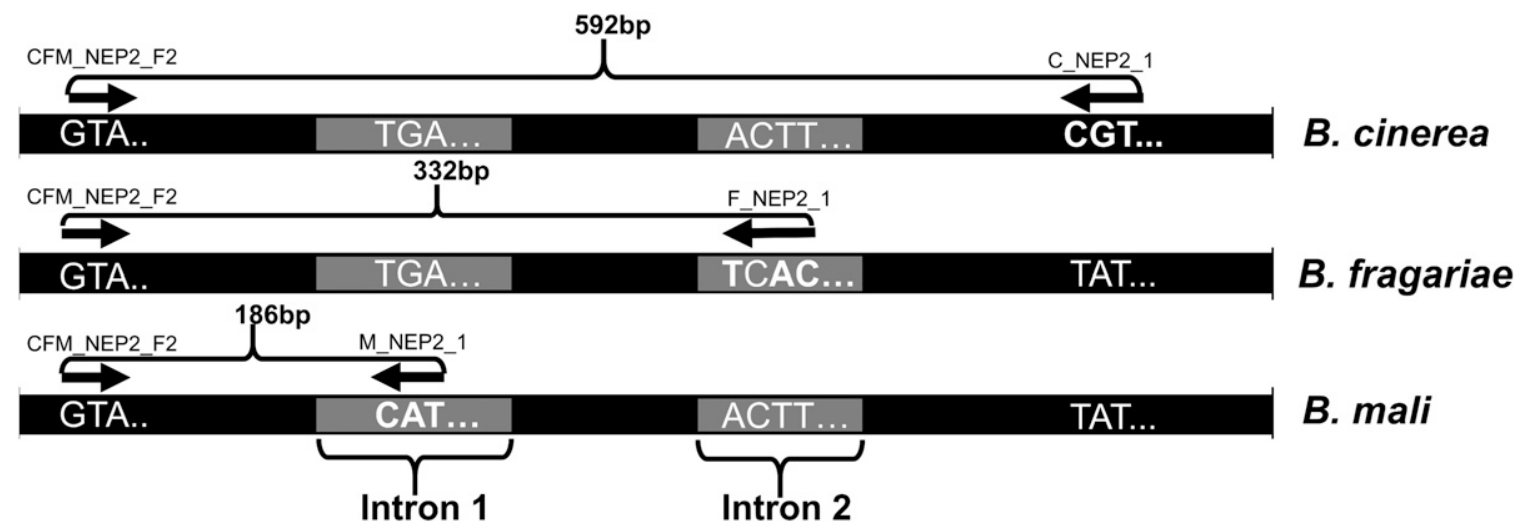

Fig. 1. Schematic of a 705-bp fragment of the NEP2 gene showing primer location and size of fragments produced by each primer set for Botrytis spp. identification. Each solid black arrow represents a primer and the name of the primer is listed above the arrow. Letters below each arrow indicate the first three or four nucleotides where each primer anneals. Nucleotides that vary between species and allow differentiation are shown in bold. Gray segments indicate introns and black segments represent exons. 
of $\alpha=0.05$ to determine whether different species truly have differing sensitivities to Polyoxin-D. Because only three $B$. mali isolates were identified and they all belonged to the RS category, these were not included in the analysis.

\section{Results}

Cultural characteristics and molecular identification of isolates. Sequencing of the $G 3 P D H$ gene was performed to identify isolates to the species level that had colony morphologies on PDA different from $B$. cinerea. These distinguishing morphological characteristics included colony color and shape as well as the isolates' ability to sporulate and produce sclerotia. BLAST search revealed that these morphologically unique isolates represented the three species $B$. cinerea, B. fragariae, and B. mali. On PDA, B. cinerea produced characteristic prolific grayish-brown spores, whereas $B$. fragariae colonies were typically white and sporulation was less obvious (Fig. 2). When B. fragariae sclerotia were present on minimal media, they typically formed concentric circles, whereas $B$. cinerea sclerotia placement was random. In rare cases, some $B$. fragariae isolates did produce grayish spores and conidiophores similar to $B$. cinerea. The three $B$. mali isolates obtained were not consistent in morphology but also did not appear to sporulate as readily as $B$. cinerea isolates.

G3PDH, RPB2, HSP60, NEP1, and NEP2 nucleotide sequence analysis of Botrytis isolates revealed high interspecific but low intraspecific variation between and among $B$. cinerea, $B$. fragariae,

Table 2. Species-specific primer sequences for distinguishing Botrytis cinerea from B. fragariae and B. mali

\begin{tabular}{lll}
\hline Name & \multicolumn{1}{c}{ Nucleotide sequence } & Anneals to \\
\hline CFM_NEP2 & 5'-GTAGGAACAGTTTATGAG-3' $^{\prime}$ & $\begin{array}{c}\text { All three } \\
\text { species }\end{array}$ \\
C_NEP2 & 5'-GACCCATTGAGTGATCGACG-3' & B. cinerea \\
F_NEP2 & 5'-TAGTTTGGATCTGTAAGGAGGTGA-3' & B. fragariae \\
M_NEP2 & 5'-ACCACTAAGAAACGTTAGAGACATG-3' & B. mali \\
\hline
\end{tabular}

and B. mali species. Pairwise comparison of sequences revealed that, within each species, there were no more than 2 nucleotide differences out of approximately $900 \mathrm{bp}$. Between species, however, there were more substantial differences (Table 3).

Development of species-specific primers. Primers were developed to differentiate the three species using NEP2 gene nucleotide sequence variability (Fig. 1). The primers yielded the expected fragment sizes of 592 bp for $B$. cinerea, $332 \mathrm{bp}$ for $B$. fragariae, and 186 bp for $B$. mali (Fig. 3). The same results were obtained when Botrytis aerial mycelium removed directly from detached fruit was used as template. However, no bands were obtained for common media and fruit contaminants Fusarium, Cladosporium, Rhizopus, and Penicillium spp. (data not shown). DNA from B. caroliniana (Fig. 3) and $B$. ricini used as templates produced no amplicons, though positive controls using $B$. caroliniana-specific primers and ITS of $B$. ricini resulted in amplification of the expected band sizes.

Overall species identification results for isolate collections. Of the 78 isolates selected for this study based on the three Polyoxin-D sensitivity phenotypes, we identified 36 B. cinerea, 39 B. fragariae, and $3 \mathrm{~B}$. mali isolates using species-specific PCR and G3PDH sequencing. Most $(n=70)$ isolates were collected from blossoms of 19 different farms, and the majority of these (58.2\%) were B. fragariae. The remaining eight isolates were from fruit collected from the same location and were all $B$. cinerea.

With regard to Polyoxin-D sensitivity, the vast majority of S isolates $(n=27 ; 96.4 \%)$ were $B$. cinerea, the majority of RS isolates $(n=$ $22 ; 78.6 \%$ ) were $B$. fragariae (Fig. 4 ), and a statistically significant relationship between species and Polyoxin-D sensitivity was detected $(P<0.0001)$. Most MS isolates $(n=16 ; 72.7 \%)$ were $B$. fragariae. All but one of the $B$. cinerea MS isolates had relative growth values below $20 \%$ on Polyoxin-D amended medium, whereas all MS B. fragariae isolates had relative growth values above $20 \%$. All 91 isolates obtained from various nurseries most likely to provide transplants to the locations we sampled were $B$. cinerea based on species-specific PCR results.

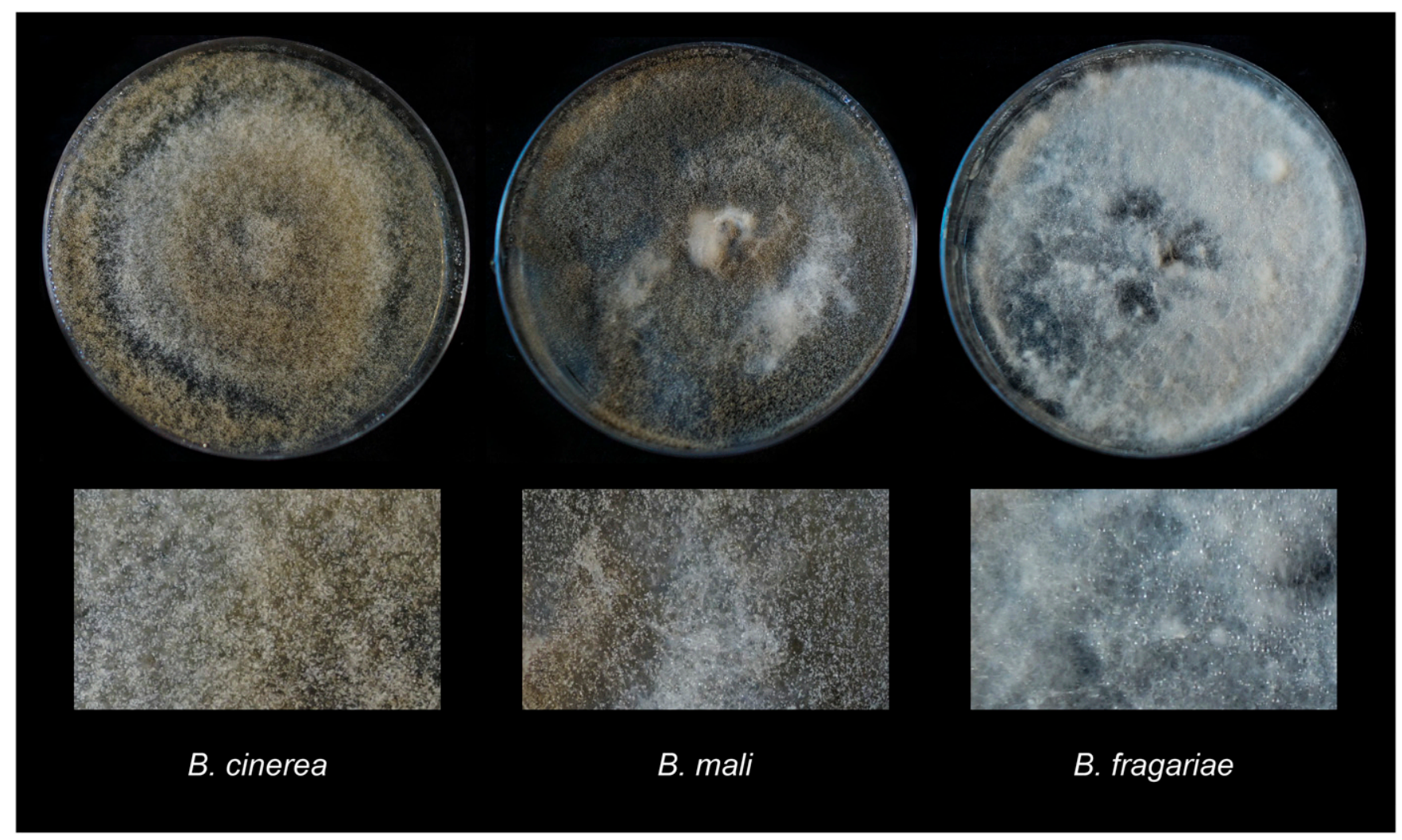

Fig. 2. Morphology in form of sporulation capacity (above) and hyphal density (below) of representative isolates of each species on potato dextrose agar after approximately 1 month of growth. Isolates 14.9.15, 36.3.15, and 13.6.15 represented the species Botrytis cinerea, $B$. mali, and B. fragariae, respectively. 
We collected 46 Botrytis isolates from a single location in Mullins, $\mathrm{SC}$, to determine host tissue preference of species. All 28 blossom isolates were $B$. fragariae as well as 6 of the 8 isolates collected from leaves (Fig. 5). All 10 isolates collected from fruit later in the season were identified as B. cinerea (Fig. 5), indicating that the two species had host tissue preference. A possible limitation of this analysis was that some leaf and fruit samples were treated with Polyoxin-D, which could potentially select for $B$. fragariae because this species is prone to RS to Polyoxin-D. However, none of the 10 fruit samples from control or Polyoxin-D-treated plots were $B$. fragariae, and several leaf samples from the untreated control block were $B$. fragariae, indicating that Polyoxin-D-induced selection for $B$. fragariae did not skew our host tissue preference results.

Table 3. Pairwise comparison showing the average percentage of each sequence that differed between species ${ }^{\mathrm{a}}$

\begin{tabular}{llccccc}
\hline & & \multicolumn{5}{c}{ Gene (\%) } \\
\cline { 3 - 7 } Species & Compared with & G3PDH & HSP60 & RPB2 & NEP1 & NEP2 \\
\hline Botrytis & B. cinerea & 4.2 & 4.4 & 4.7 & 9.6 & 1.2 \\
fragariae & & & & & & \\
B. cinerea & B. mali & 1.4 & 3.0 & 4.4 & N/A & 1.2 \\
B. mali & B. fragariae & 3.8 & 4.7 & 2.8 & N/A & 9.6 \\
\hline
\end{tabular}

a Percentage was obtained by dividing the number of nucleotide differences between species by the total sequence length. N/A $=$ NEP1 gene sequence was not obtained for $B$. mali.

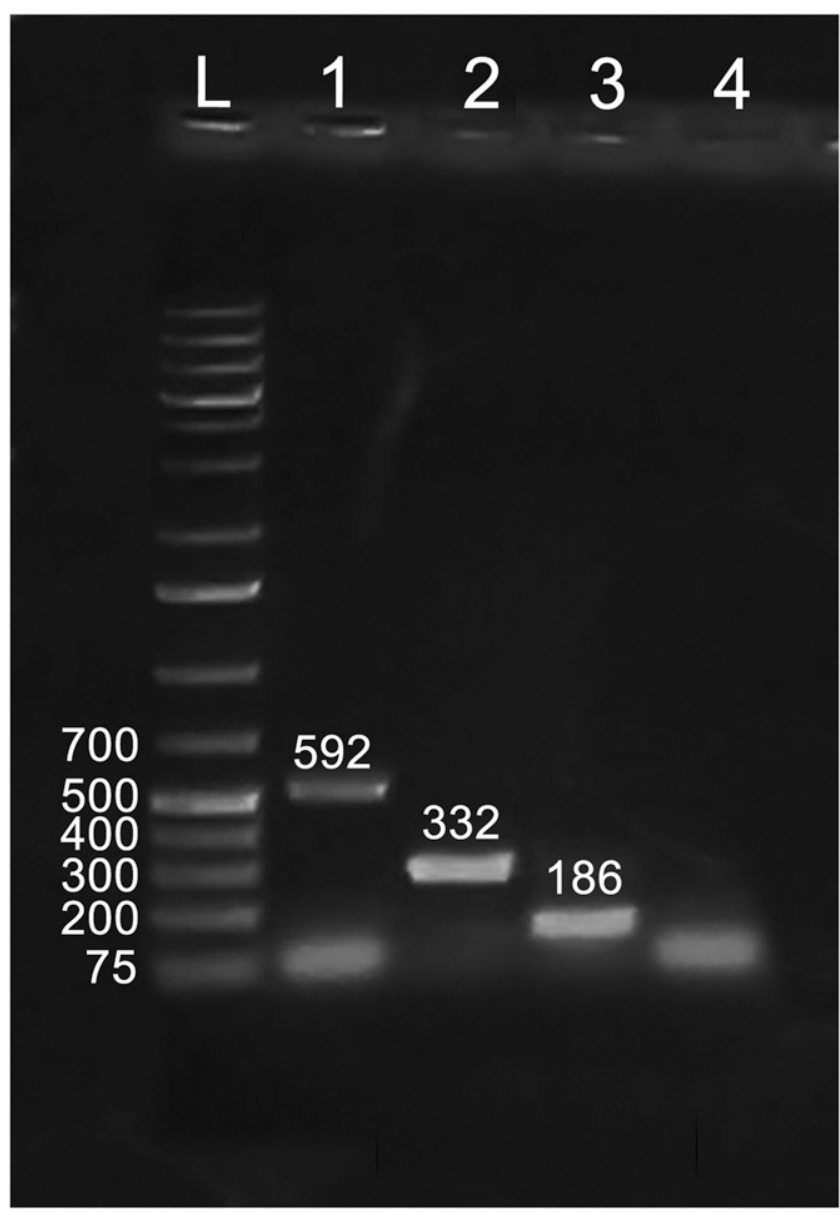

Fig. 3. Amplification of Botrytis spp. collected from strawberry using species-specific primers CFM_NEP2, C_NEP2, F_NEP2, and M_NEP2. Lanes 1, 2, and 3 contain polymerase chain reaction (PCR) products amplified from $B$. cinerea, $B$. fragariae, and $B$. mali templates, respectively. Lane 4 contains no amplicon from an attempt to amplify from a $B$. caroliniana template. Lane $L=$ GeneRuler 1-kb Plus DNA Ladder (Thermo Fisher Scientific, Waltham, MA). B. caroliniana, B. ricini (data not shown), and failed PCR produced no amplicons but leftover PCR materials are visible in the bottom of the lane and may appear as a band, as seen in lanes 1 and 4 .

\section{Discussion}

In a previous study, $B$. fragariae was reported, for the first time, to exist in the United States (Rupp et al. 2017). These isolates were from three commercial farms in South Carolina. The results of the present study show that $B$. fragariae occurs in at least two more states (North Carolina and Ohio), indicating that this species may be widely distributed in the United States. B. fragariae was also detected in Germany at low frequencies in four different states (Rupp et al. 2017). However, our sample size and collection bias to represent equal numbers of Polyoxin-D sensitivity phenotypes does not allow for an evaluation of frequency of $B$. fragariae in U.S. strawberry fields. B. caroliniana, a species isolated in 2011 from a single strawberry fruit, and B. ricini, detected in 2013 in Florida, were not found in our sample populations (Amiri et al. 2016; Li et al. 2012). Species-specific primers were designed that effectively distinguished isolates of B. cinerea, B. mali, and B. fragariae. Approximately $10 \%$ of the isolates did not produce any amplicon when these primers were used. It is possible that these isolates did not contain the introns that the species-specific primers are based on.

B. fragariae isolates were more likely $(P<0.0001)$ to be RS to Polyoxin-D compared with $B$. cinerea. The few $B$. fragariae and $B$. cinerea isolates not following this observation are likely outliers of a normal baseline sensitivity distribution. Innate RS to Polyoxin-D may be caused by constitutive expression of drug efflux pumps, resulting in a higher baseline sensitivity to Polyoxin-D in $B$. fragariae compared with $B$. cinerea. This hypothesis is supported by evidence

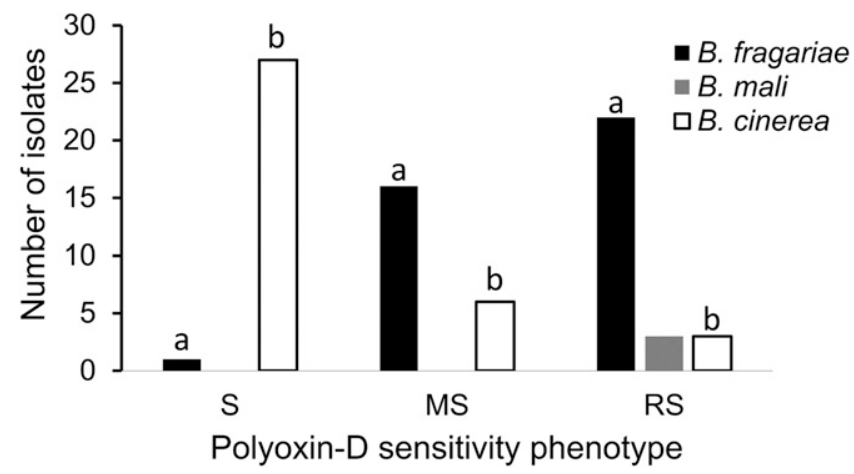

Fig. 4. Number of isolates collected from flowers in multiple states and locations within states for three Botrytis spp. sensitive (S; $0 \%$ relative growth), moderately sensitive (MS; $>0$ to $70 \%$ relative growth), and reduced sensitive (RS; $>70 \%$ relative growth) to Polyoxin-D. Letters representing statistical differences are valid within each phenotype but not across phenotypes. The number of locations refers to the number of farms where isolates were collected. $S$ isolates were found in all five states (15 locations), MS in four states (12 locations), and RS isolates in three states (13 locations).

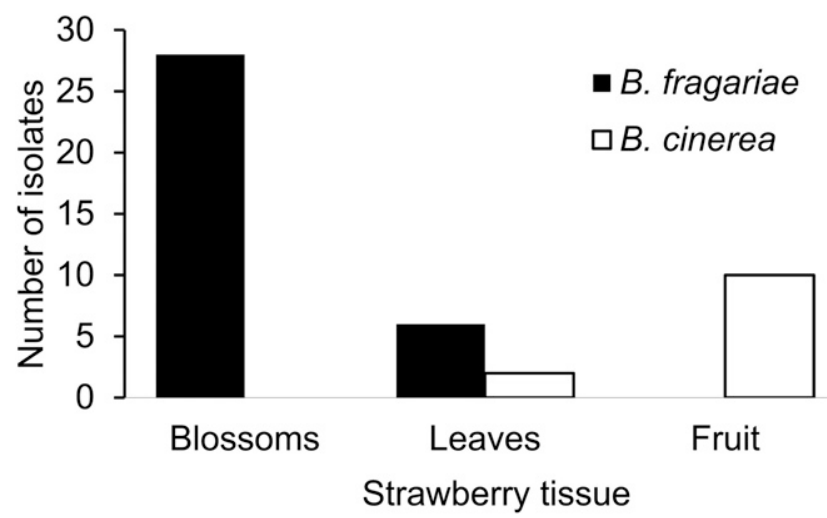

Fig. 5. Species distribution of Mullins, SC isolates collected from different strawberry tissues preharvest (blossoms), midharvest (leaves), and postharvest (fruit) to determine whether Botrytis fragariae had preference for blossom tissue. 
that $B$. fragariae isolates from German strawberry fields have higher basal expression levels of $a t r B$ than equivalent $B$. cinerea isolates (Rupp et al. 2017). However, whether or not Polyoxin-D is a substrate of $\operatorname{atr} B$ is not known. B. fragariae may constitutively express other drug efflux genes such as the $B M R l$ gene encoding an $\mathrm{ABC}$ transporter previously correlated with Polyoxin-D efflux at levels higher than B. cinerea isolates (Nakajima et al. 2001). Another possibility is that chitin synthetase enzymes of $B$. fragariae and $B$. cinerea differ in amino acid sequence, which may result in differential binding affinity to Polyoxin-D in the two Botrytis spp. This type of differential binding affinity resulting in sensitivity differences has already been observed in the pathogen Mycosphaerella graminicola, where amino acid sequence differences in the enzyme MgCYP51 may result in decreased binding affinities and reduced sensitivity to demethylation inhibitor (DMI) fungicides (Mullins et al. 2011). All three B. mali isolates were RS to Polyoxin-D but the sample size was too small to allow for solid conclusions about innate RS to Polyoxin-D in this species.

Though B. fragariae is present in several states of Germany and the United States, the source of $B$. fragariae inoculum is unknown. The isolates used in this study came from plasticulture fields with raised beds that were fumigated prior to planting nursery transplants in the fall. Therefore, it is reasonable to hypothesize that $B$. fragariae may have come from nurseries, especially because spread of $B$. cinerea inoculum from nursery to field through latent infections of nursery stock is well documented (Oliveira and Peres 2014; Peres 2015; Schnabel et al. 2015). Screening of 91 isolates from major nursery stock providers yielded only $B$. cinerea. Because all nursery isolates were obtained from symptomatic leaves, whereas most isolates used for Polyoxin-D sensitivity profiling came from blossoms, a host tissue preference could explain our inability to find $B$. fragariae in nursery stock. Results from a commercial farm in Mullins, SC, indicated that B. fragariae prefers blossoms to either leaves or fruit but both species were found in leaves. This indicates that host tissue preference is not the best explanation for the absence of $B$. fragariae in nursery isolates. Though B. fragariae was found on leaves in Mullins, SC, it was not found on fruit. In fact, a complete species shift occurred: all isolates recovered from blossoms were $B$. fragariae and all isolates recovered from fruit on the same plants were $B$. cinerea (Fig. 5). Subsequent analysis of our collection from Clemson did reveal instances of $B$. fragariae infecting fruit tissue, but they were rare (data not shown). These results agree with competition studies performed in Germany which revealed that B. fragariae is outcompeted by B. cinerea on fruit in vitro (Rupp et al. 2017).

Because no $B$. fragariae was found in a collection from nursery stock, inoculum may instead be endemic and originate from overwintering plant debris or alternate hosts near strawberry plantings. Because we found that Polyoxin-D RS isolates produce more sclerotia than S isolates in vitro, overwintering of $B$. fragariae sclerotia between planting beds in the form of sclerotia appears more plausible, especially because $B$. fragariae appears to have a limited host range (Dowling et al. 2016; Rupp et al. 2017).

In conclusion, B. fragariae from U.S. strawberry fields differs from $B$. cinerea in sensitivity to Polyoxin-D, cultural characteristics on PDA medium, inoculum source, and host tissue preference. The newly developed primers reported in this study will allow for easy distinction between the species causing gray mold of strawberry.

\section{Acknowledgments}

This project was supported by Technical Contribution Number 6525 of the Clemson University Experiment Station and by the United States Department of Agriculture National Institute of Food and Agriculture under project number SC-1700501 and Specialty Crop Research Initiative grant number 2014-5118122377. We thank C. J. Porter, J. Ballew, N. Peres, M. Oliviera, and A. Zuniga for providing some of the isolates for this study; and K. Bryson, B. Cox, and T. Sroka for technical assistance.

\section{Literature Cited}

Amiri, A., Onofre, R. B., and Peres, N. A. 2016. First report of gray mold caused by Botryotinia ricini (Amphobotrys ricini) on strawberry in United States. Plant Dis. 100:1007.

Chilvers, M. I., and du Toit, L. J. 2006. Detection and identification of Botrytis species associated with neck rot, scape blight, and umbel blight of onion. Plant Health Prog. doi:10.1094/PHP-2006-1127-01-DG

Dowling, M. E., Hu, M.-J., Schmitz, L. T., Wilson, J. R., and Schnabel, G. 2016 Characterization of Botrytis cinerea isolates from strawberry with reduced sensitivity to Polyoxin D zinc salt. Plant Dis. 100:2057-2061.

Dowling, M. E., and Schnabel, G. 2017. First report of Botrytis mali causing gray mold on strawberry in the United States. Plant Dis. 101:1034.

Fillinger, S., and Elad, Y. 2015. Botrytis-The Fungus, the Pathogen and its Management in Agricultural Systems. Springer, Cham, Switzerland.

Fournier, E., Giraud, T., Albertini, C., and Brygoo, Y. 2005. Partition of the Botrytis cinerea complex in France using multiple gene genealogies. Mycologia 97: 1251-1267.

Li, X., Fernández-Ortuño, D., Chai, W., Wang, F., and Schnabel, G. 2012 Identification and prevalence of Botrytis spp. from blackberry and strawberry fields of the Carolinas. Plant Dis. 96:1634-1637.

Montone, V. O., Fraisse, C. W., Peres, N. A., Sentelhas, P. C., Gleason, M., Ellis M., and Schnabel, G. 2016. Evaluation of leaf wetness duration models for operational use in strawberry disease-warning systems in four US states. Int. J. Biometeorol. 60:1761-1774.

Mullins, J. G. L., Parker, J. E., Cools, H. J., Togawa, R. C., Lucas, J. A., Fraaije, B. A., Kelly, D. E., and Kelly, S. L. 2011. Molecular modelling of the emergence of azole resistance in Mycosphaerella graminicola. PLoS One 6:e20973.

Nakajima, M., Suzuki, J., Hosaka, T., Tadaaki, H., and Akutsu, K. 2001 Functional analysis of an ATP-binding cassette transporter gene in Botrytis cinerea by gene disruption. J. Gen. Plant Pathol. 67:212-214.

Oliveira, M. S., and Peres, N. A. 2014. Strawberry nursery plants as a source of Botrytis cinerea isolates resistant to fungicides. Poster 208-P in: 2014 American Phytopathological Society Annual Meeting, Minneapolis, MN

Peres, N. A. 2015. 2015 Florida Plant Disease Management Guide: Strawberry. Online publication. University of Florida. http://edis.ifas.ufl.edu/pg056

Plesken, C., Weber, R. W. S., Rupp, S., Leroch, M., and Hahn, M. 2015. Botrytis pseudocinerea is a significant pathogen of several crop plants but susceptible to displacement by fungicide-resistant B. cinerea strains. Appl. Environ. Microbiol. 81:7048-7056.

Rupp, S., Plesken, C., Rumsey, S., Dowling, M., Schnabel, G., Weber, R. W. S., and Hahn, M. 2017. Botrytis fragariae, a new species causing gray mold on strawberries, shows high frequencies of specific and efflux-based fungicide resistance. Appl. Environ. Microbiol. 83:e00269-17.

Schnabel, G., Hu, M., and Fernández-Ortuño, D. 2015. Monitoring resistance by bioassay: Relating results to field use using culturing methods. Pages 281-293 in: Fungicide Resistance in Plant Pathogens. H. Ishii and D. W. Hollomon, eds. Springer, Tokyo.

Staats, M., van Baarlen, P., Schouten, A., van Kan, J. A. L., and Bakker, F. T. 2007. Positive selection in phytotoxic protein-encoding genes of Botrytis species. Fungal Genet. Biol. 44:52-63.

Staats, M., van Baarlen, P., and van Kan, J. A. L. 2005. Molecular phylogeny of the plant pathogenic genus Botrytis and the evolution of host specificity. Mol. Biol Evol. 22:333-346.

Walker, A.-S., Gautier, A., Confais, J., Martinho, D., Viaud, M., Le Pêcheur, P., Dupont, J., and Fournier, E. 2011. Botrytis pseudocinerea, a new cryptic species causing gray mold in French vineyards in sympatry with Botrytis cinerea. Phytopathology 101:1433-1445. 